\title{
Aberrant origin of the right coronary artery as a potential cause of sudden death: successful anatomical correction
}

\author{
C Nelson-Piercy, A F Rickards, M H Yacoub
}

\begin{abstract}
A man with an aberrant right coronary artery and haemodynamically important prolapse of the mitral valve was successfully resuscitated. The aberrant right coronary artery was thought to be a possible cause of the cardiopulmonary arrest in this patient. Both lesions were corrected at a single operation.
\end{abstract}

Some believe that the risk of sudden death is higher in patients in whom the right coronary artery arises aberrantly from the left sinus of Valsalva. ${ }^{1}$ Many cases of sudden death associated with aberrant left coronary arteries have been reported, ${ }^{2-4}$ but sudden death secondary to an anomalous right coronary artery is less well recognised..$^{5-8}$ Mitral valve prolapse too predisposes to fatal arrhythmic events. $^{9-11}$

We describe the resuscitation and operative treatment of a patient with an anomalous right coronary artery and mitral valve prolapse.

\section{Case report}

A 67 year old man was admitted to hospital after he was resuscitated from a cardiorespiratory arrest that occurred while he was asleep at home.

A heart murmur was detected 16 years before. There was no history of rheumatic fever. In July 1988 mitral valve prolapse and mitral regurgitation secondary to ruptured chordae tendineae were diagnosed after a six month history of exertional dyspnoea and palpitation. In November 1988 fast atrial fibrillation was first noted and the patient was treated with digitalis. Serial electrocardiograms and echocardiograms suggested increasing left ventricular and left atrial dimensions, but the symptoms did not worsen.

During the night of 18 August 1989 the patient's wife (an ex-nurse) was woken by the sound of her husband groaning. She found that he was cyanosed, pulseless, and unresponsive and she started external cardiac massage and mouth to mouth resuscitation. She continued for 20 minutes until an ambulance with a defibrillator arrived. The patient was treated with two DC shocks and with lignocaine and atropine and he regained consciousness. Presumed ventricular fibrillation was converted to fast atrial fibrillation.

When he arrived at hospital he had an irregular pulse of 124 beats per minute. The jugular venous pressure was elevated and the blood pressure was $100 / 70 \mathrm{~mm} \mathrm{Hg}$. The apical cardiac impuse was displaced laterally and there was a grade $4 / 6$ pansystolic murmur at the apex, radiating into the axilla, and an associated thrill between the apex and lower left sternal edge. Auscultation of the chest detected bilateral basal crackles.

The concentration of serum potassium was $3.7 \mathrm{mmol} / 1$ and serum digoxin was $0.87 \mathrm{ng} / \mathrm{ml}$. The electrocardiogram confirmed atrial fibrillation with multifocal ventricular extrasystoles, left ventricular hypertrophy, and a strain pattern. The chest $x$ ray showed cardiomegaly with an enlarged left atrium and diversion of blood to the upper lobes. Echocardiography showed a dilated left ventricle (end systolic dimension $5.5 \mathrm{~cm}$ ) with good contraction and a dilated left atrium (5.6 $\mathrm{cm})$. There was prolapse of the posterior leaflet of the mitral valve and ruptured chordae were seen flicking into the left atrium (fig 1A). Doppler echocardiography confirmed considerable mitral regurgitation. Cardiac catheterisation showed a pulmonary artery wedge pressure of $16 \mathrm{~mm} \mathrm{Hg}$. Pulmonary artery pressure was $80 / 30$, right ventricular pressure $70 / 4$, and mean right atrial pressure $6 \mathrm{~mm} \mathrm{Hg}$. The aortic pressure was 100/70 $\mathrm{mm} \mathrm{Hg}$. Left ventricular pressure was 100 with an end diastolic pressure of $16 \mathrm{~mm} \mathrm{Hg}$. Cineangiography showed a dilated and hyperkinetic left ventricle with grade IV mitral regurgitation. At coronary angiography the right coronary ostium could not be intubated but aortic root injection suggested that the right coronary artery originated from the left coronary sinus (fig 1B). The origin and course of the left coronary artery were normal. Treatment with diuretics and oral digoxin stabilised the patient's condition.

\section{OPERATION}

At operation, performed on 5 September 1989 with cardiopulmonary bypass, the surgeon found an enlarged left atrial cavity, and a typically floppy mitral valve with prolapse of a large segment of the posterior and medial cusps caused by rupture of three major chordae. Elongated chordae caused a partial prolapse of the anterior cusp. Inspection of the coronary ostia from within the aortic root showed that the right coronary artery originated from the left coronary sinus. The right coronary orifice was situated just posterior to the free edge of the intercoronary commissure. The proximal part of the right coronary artery ran in the wall of the aorta to the midpoint of the right coronary sinus. There- 
Figure 1 ( $A$ )

Echocardiogram showing the dilated left atrium and prolapse of the posterior leaflet of the mitral valve. Ruptured chordae were seen flicking into a dilated left atrium. $L V$; left ventricle; $L A$, left atrium; PLMV, posterior leaflet of mitral valve; $\mathrm{CH}$, ruptured chordae. (B) Aortogram showing normal origin of the left main coronary artery from the left coronary sinus. The right coronary artery originated aberrantly from the left coronary sinus. $L M C A$, left main coronary artery; $R C A$, right coronary artery.
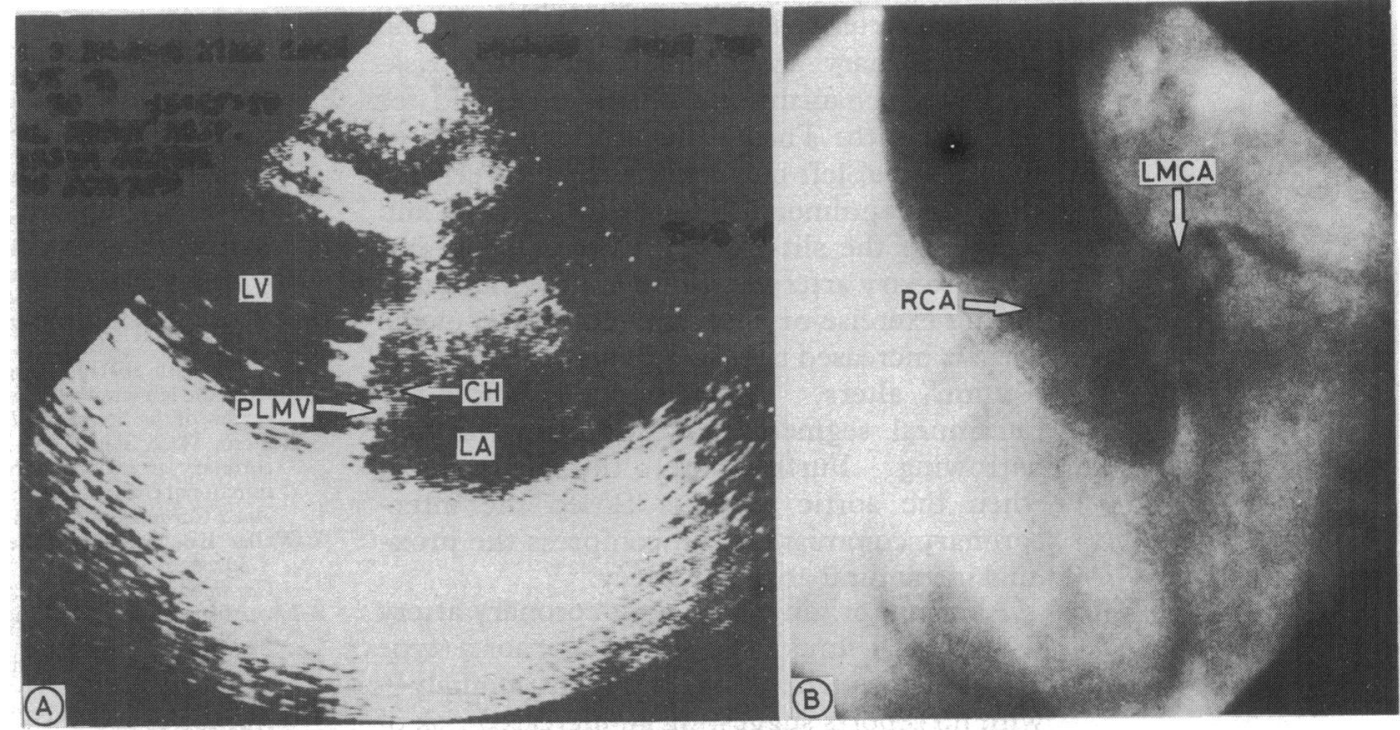

after its course was normal (fig 2A). The mitral valve was repaired and the aberrant origin of the right coronary artery was corrected by a technique similar to that used by us to correct an aberrant left coronary artery. ${ }^{12}$ The intramural part of the right coronary artery" was deroofed (by excising the inner aortic wall) as far as the midpoint of the right coronary sinus. This resulted in prolapse of the intercoronary commissure. The intimal flap of the coronary artery was sutured to the intima of the aorta and the top of the intercoronary commissure was reattached to the aortic wall. This transposed the aberrant
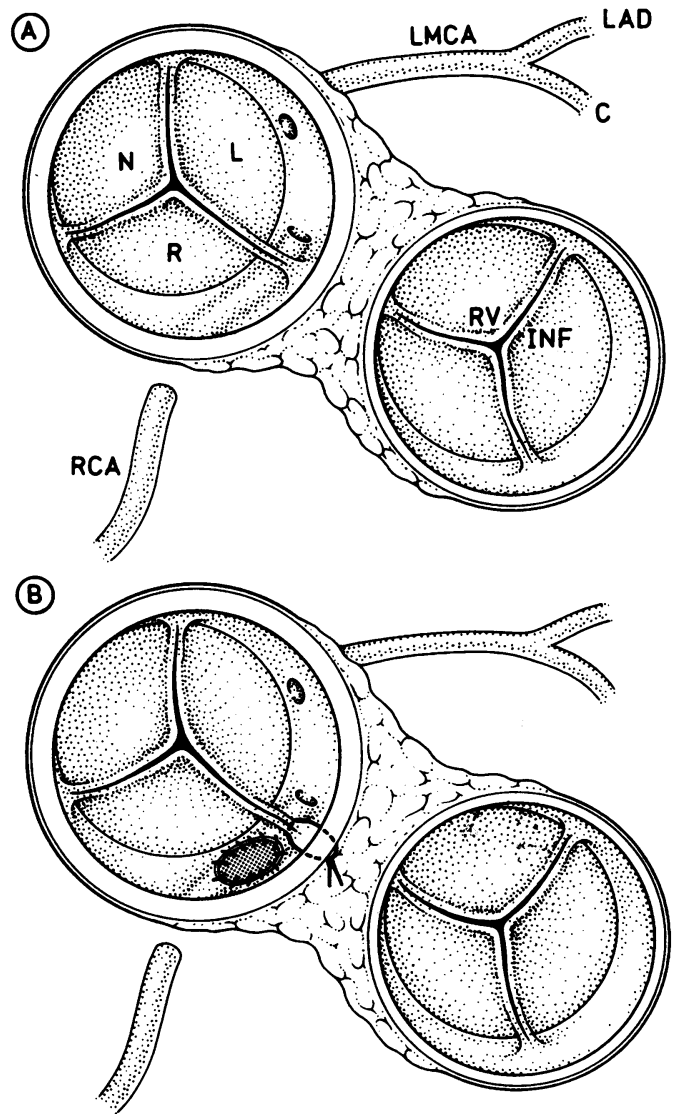

coronary artery to its normal position (fig 2B).

The postoperative course was uneventful. The patient remained in sinus rhythm for two days, but subsequently reverted to atrial fibrillation; there was no recurrence of the ventricular extrasystoles seen preoperatively. A postoperative echocardiogram showed a smaller left atrial cavity $(3 \cdot 8 \mathrm{~cm})$.

\section{Discussion}

Mitral valve prolapse is associated, albeit rarely, with sudden death. Death is probably caused by arrhythmia. ${ }^{91013}$ Kligfield et al also reported depressed myocardial function in patients with mitral regurgitation (with or without mitral valve prolapse) who died suddenly. ${ }^{11}$ The complexity of arrhythmia in mitral regurgitation seems to be independent of its aetiology. ${ }^{10}$ Barlow and Pocock have suggested several mechanisms by which floppy leaflets and elongated chordae could predispose to ventricular extrasystoles. ${ }^{13}$ These include endocardial lesions produced by contact between the chordae and left venpapillary muscles by the voluminous leaflets.

Thus sudden death in patients with mitral valve prolapse with considerable mitral regurgitation may be related to the haemodynamic and arrhythmogenic consequences of mitral regurgitation or to mitral valve prolapse itself. Mitral valvoplasty was successful in a patient with mitral valve prolapse and potentially lethal ventricular arrhythmias but without haemodynamically important mitral regurgitation (J B Barlow, personal communication).

Anomalous patterns of origin of the coronary arteries were classified by Vlodaver et al. ${ }^{14}$ Aberrant origin of the left coronary artery from the right aortic sinus (type II) was shown to be associated with sudden death during exertion. Most reported cases were in young men often with a history suggestive of antecedent episodes of ischaemia, although cardiac arrest can be the first presentation. Patients in whom the proximal left main tricular endocardium, and traction on the 
coronary artery runs between the aorta and the pulmonary artery are at particular risk. Several mechanisms have been suggested for sudden death. These include compression of the proximal left coronary artery between the aorta and pulmonary artery, ${ }^{4}$ kinking, ${ }^{2}$ and closure of the slit-like orifice of the aberrant left coronary artery by a flap. ${ }^{3}$ We believe that during exercise or whenever expansion of the aorta is increased the angle of the left coronary ostium alters, severely stretching the intramural segment and producing luminal narrowing. Furthermore, during diastole when the aortic valve is closed the intercoronary commissure can compress the proximal intramural aberrant artery. ${ }^{12} 15$

Aberrant origin of the right coronary artery from the left sinus of Valsalva of the aorta (type I) was not thought to be a dangerous anomalywith no reports suggesting an increased risk of sudden death. In 1982, however, a necropsy review of 10 patients with this anomaly showed that three had died suddenly. ${ }^{6}$ In 1985 Waller reported the death during exertion of a 17 year old boy with origin of the right coronary artery from the left sinus of Valsalva. ${ }^{7}$ Two further cases were reported by Liberthson in $1983^{5}$ and $1989 .^{8}$ The latter patient survived an episode of ventricular fibrillation and subsequently underwent successful bypass grafting with an internal mammary artery. Surgical correction and coronary bypass grafting have been described as methods of preventing sudden death in patients with aberrant left coronary arteries. ${ }^{121516}$ We suggest that compression of the intramural part of the coronary artery is the major cause of sudden ischaemia when the origin of either the left or the right coronary artery is abnormal.
1 Liberthson RR, Dinsmore RE, Fallon JT. Aberrant coronary artery origin from the aorta. Report of 18 patients review of literature and delineation of natural history and management. Circulation 1979;59:748-54.

2 Chaitman BR, Lesperance J, Saltiel J, Bourassa MG Clinical, angiographic, and haemodynamic findings in patients with anomalous origin of the coronary arteries. Circulation 1976;53:122-30.

3 Cheitlin MD, De Castro CM, McAllister HA. Sudden death as a complication of anomalous left coronary origin from

4 Benson PA, Lack AR. Anomalous aortic origin of the left coronary artery. Arch Pathol 1968;86:214-6.

5 Liberthson RR, Gang DL, Custer J. Sudden death in an infant with aberrant origin of the right coronary artery from the left sinus of Valsalva of the aorta: case report and review of the literature. Pediatr Cardiol 1983;4:45-8.

6 Roberts WC, Siegel RJ, Zipes DP. Origin of the right coronary artery from the left sinus of valsalva and its functional consequences: analysis of 10 necropsy patients. Am J Cardiol 1982;49:863-8.

7 Waller BF. Exercise-related sudden death in young (age $<30$ years) and old (age $>30$ years) conditioned subjects. Cardiovasc Clin 1985;15:9-73.

8 Liberthson RR. Discussion in: Scully RE, Mark EJ, McNeely WF, McNeely BU. Case records of the Massachusetts General Hospital. $N$ Engl J Med 1989;320 1475-83.

9 Jeresaty RM. Mital valve prolapse. New York: Raven Press 1979:209-21.

10 Kligfield P, Hochreiter C, Kramer $\mathrm{H}$, et al. Complex arrhythmias in mitral regurgitation with and without mitral valve prolapse: contrast to arrhythmias in mitral valve prolapse without mitral regurgitation. Am J Cardio 1985;55:1545-9.

11 Kligfield P, Hochreiter C, Niles N, Devereux RB, Borer JS. Relation of sudden death in pure mitral regurgitation, with and without mitral valve prolapse, to repetitive ventricular arrhythmias and right and left ventricular ejection fractions. Am J Cardiol 1987;60:397-9.

12 Mustafa I, Gula G, Radley-Smith R, Durrer S, Yacoub MH Anomalous origin of the left coronary artery from the anterior aortic sinus: a potential cause of sudden death. $J$ Thorac Cardiovasc Surg 1981;82:297-300.

13 Barlow JB, Pocock WA. Mitral valve billowing and prolapse: perspective at 25 years. Herz 1988;13:227-34.

14 Vlodaver Z, Neufeld HN, Edwards JE. Coronary arterial variations in the normal heart and in congenital heart disease. New York: Academic Press, 1975:23-35.

15 Donaldson RM, Raphael MJ, Yacoub MH, Ross DN. Haemodynamically significant anomalies of the coronary arteries. Surgical aspects. Thorac Cardiovasc Surg 1982;30:7-13

16 Moodie DS, Gill C, Loop FD, Sheldon WC. Anomalous left main coronary artery originating from the right sinus of valsalva. Pathophysiology, angiographic definition and surgical approaches. J Thorac Cardiovasc Surg 1980;
80:198-205. 